\title{
node-indri: moving the Indri toolkit to the modern Web stack
}

\author{
Felipe Moraes and Claudia Hauff \\ Delft University of Technology \\ $\{$ f.moraes, c.hauff $\}$ @tudelft.nl
}

\begin{abstract}
We introduce node-indri, a Node.js module that acts as a wrapper around the Indri toolkit, and thus makes an established IR toolkit accessible to the modern web stack. node-indri exposes many of Indri's functionalities and provides direct access to document content and retrieval scores for web development (in contrast to, for instance, the Pyndri wrapper). This setup reduces the amount of glue code that has to be developed and maintained when researching search interfaces, which today tend to be developed with specific JavaScript libraries such as React.js, Angular.js or Vue.js. The node-indri repository is opensourced at https://github.com/felipemoraes/node-indri.
\end{abstract}

\section{Introduction}

The information retrieval (IR) field is aided by numerous efficient search engine implementations, aimed at research and industry, such as Indri [1], Lucene ${ }^{1}$, Terrier [2] and Anserini [3]. In the data science field, some of these efforts have evolved into frameworks such as Elasticsearch ${ }^{2}$ and Terrier-Spark [4]. Recently, in order to enable data scientists to make use of Indri as part of their workflow, Van Gysel et. [5] have made Indri accessible to the Python ecosystem (via Pyndri).

In this paper, we make Indri accessible to the modern web stack. Many modern web applications and frameworks make use of Node.js. ${ }^{3}$ A significant advantage of this framework is the single programming language on the client and server-side (JavaScript), which simplifies development; in addition, Node.js is highly scalable [6]. In order to design and evaluate web search interfaces, a backend, implemented in Node.js, requires access to a search system. One option is to call Indri via system calls. However, the disadvantage of system calls via shell commands is the extra layer of communication with the operating system.

Here, we present an alternative, node-indri, a Node.js module implemented with an easy-to-use API. It provides access to basic Indri functionalities such as search with relevance feedback and document scoring. Importantly, node-indri is implemented in a non-blocking manner. We here discuss node-indri's module

\footnotetext{
${ }^{1}$ http://lucene.apache.org/

2 https://www.elastic.co/products/elasticsearch

${ }^{3}$ https://nodejs.org/
} 
features and we compare its efficiency with Indri and Pyndri on the Aquaint and ClueWeb12 corpora with a load of $10 \mathrm{~K}$ queries. We find that node-indri can be efficiently used in modern web backend development with comparable efficiency to Indri and Pyndri.

\section{The node-indri module}

node-indri's development started with the need to make Indri's state-ofthe-art relevance feedback models accessible to students, that (i) tend to have little experience with $\mathrm{C}++$, but are familiar with modern web programming paradigms and (ii) are not IR experts and thus struggle to make sense of Indri's internals.

\subsection{Functionalities}

Figure 1 shows the three layers of abstraction of node-indri. Our module exposes Indri features through the Searcher, Reader, and Scorer classes. These classes are implemented in $\mathrm{C}++$ with the help of Native Abstractions for Node. $\mathrm{js}^{4}$, a series of macros that abstract away the differences between the V8 and libuv API versions (which together form the core of the Node. js framework and are written in $\mathrm{C}++$ ).

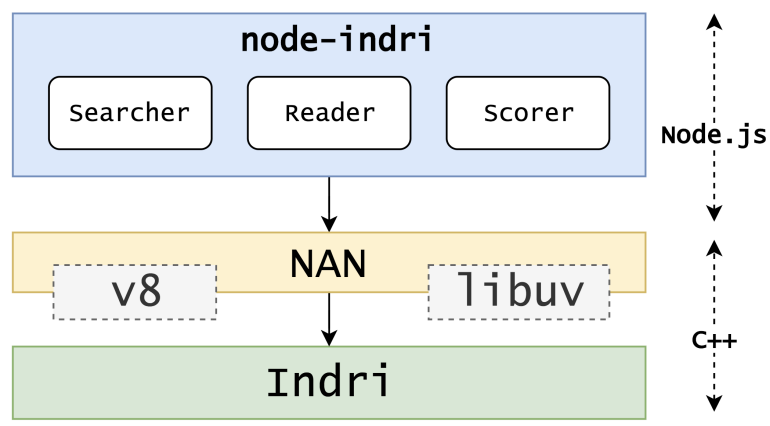

Fig. 1. Overview of node-indri layers of abstractions and their implementation languages and platforms.

In Table 1, we list the arguments of node-indri's three classes. Each class has at most two methods with arguments that depend on the functionalities exposed from Indri. The last argument is a callback function implementing the error-first pattern. In this manner, node-indri is an asynchronous module, with most of these functions assessing lower-level system functionalities through libuvl. This in turn means that the methods are executed in Node.js' thread pool, making node-indri naturally parallel.

\footnotetext{
4 https://github.com/nodejs/nan
} 
Table 1. Overview of the arguments necessary for node-indri's method calls. Our API is simple and includes only one method per class. The last argument is always a callback that is executed when the data has been retrieved. Underlined are the required parameters.

\begin{tabular}{llll}
\hline Searcher.search & Reader.getDocument & Scorer.scoreDocuments & Scorer.retrieveTopKScores \\
\hline $\begin{array}{l}\text { query, page, results- } \\
\text { per-page, feedback- } \\
\text { docs, callback }\end{array}$ & $\underline{\text { docid, callback }}$ & $\underline{\text { query, }, \underline{\text { docs}, ~} \underline{\text { callback }}}$ & $\underline{\text { query, }} \begin{array}{c}\text { number-of- } \\
\text { results, callback }\end{array}$ \\
\hline
\end{tabular}

The models' hyperparameter settings (e.g. $\mu$ in the case of language modeling with Dirichlet smoothing) are manually set via a configuration file. We now discuss the goal of each of the three classes node-indri makes available to its users in turn:

Searcher This class exposes the functionalities of Indri's QueryEnvironment and RMExpander classes through the method search which returns a list of search results in a paginated manner. When a Searcher object is instantiated, it takes a configuration object as argument (these settings include the retrieval models' hyperparameters and flags of the type of data to return). When a call to $\operatorname{search}()$ is made and no feedback documents are provided as argument, the standard query likelihood model is employed, otherwise RM3 is [7]. Depending on the configuration settings, the returned result list may contain document snippets (as provided by Indri's SnippetBuilder), document scores, document text and other metadata.

Reader This class exposes the functionalities of an Indri index through the method getDocument in order to return a document's meta- and text data.

Scorer This class provides access to the retrieval scores of a list of documents via the method scoreDocuments. In addition, it provides retrieveTopKScores to retrieve the scores and document ids of the top ranked documents for a query.

\subsection{Use Cases}

We have used node-indri as search results' provider in the backend of a largescale collaborative search system, SearchX [8]. SearchX's backend supports the inclusion of many IR backends such as Elasticsearch and Bing API calls. In order to include node-indri as one of the supported backends, we implemented Searcher to provide search results (with or without snippets) in a pagination manner leveraging feedback documents, Reader to enable the rendering of a document's content when a user has clicked on it, and Scorer to enable our backend to have direct access to documents' scores for reranking purposes.

\section{Efficiency Analysis}

We now present an efficiency analysis of node-indri, comparing it to Indri and Pyndri. We indexed two standard test corpora-Aquaint and ClueWeb12B- 
with Indri and measured the execution time for 10k queries of the TREC 2007 Million Query track ${ }^{5}$ across the three toolkits. As retrieval model we fixed language modeling with Dirichlet smoothing; up to 1000 documents were returned per query. We use Indri's IndriRunQuery application for this purpose; for Pyndri and node-indri we implemented scripts to achieve the same behaviour. Specifically, Pyndri's results were obtained with a Python script implemented with multiprocessing. In node-indri, we make use of Promises.all. We limit the execution to 20 threads for all three toolkits ${ }^{6}$. Table 2 presents the overall query execution time of the three toolkits.

Our results show that node-indri has execution times comparable to Indri and Pyndri. We can thus use node-indri efficiently in modern web backend development. We find for a small collection such as Aquaint (1 million documents), all three toolkits to obtain very similar execution times (between 25 and 29 seconds). In contrast, for a larger collection such as ClueWeb12B (50 million documents), the execution times differ to some extent: Indri takes on average 27 minutes to process $10 \mathrm{~K}$ queries, while node-indri and Pyndri take 34 and 37 minutes respectively. This is expected, as both node-indri and Pyndri have additional overhead due to the frameworks they are built upon.

Table 2. Overview of retrieval efficiency (in seconds) across two corpora. 10K queries from the TREC 2017 Million Queries track were executed 20 times. Reported are the average (standard deviation) execution time of the batches.

\begin{tabular}{lll}
\hline & Aquaint & ClueWeb12B \\
\hline Indri & $29 \mathrm{~s}(0.30 \mathrm{~s})$ & $1645 \mathrm{~s}(20 \mathrm{~s})$ \\
Pyndri & $25 \mathrm{~s}(1.22 \mathrm{~s})$ & $2262 \mathrm{~s}(340 \mathrm{~s})$ \\
\hline node-indri & $25 \mathrm{~s}(0.58 \mathrm{~s})$ & $2058 \mathrm{~s}(338 \mathrm{~s})$ \\
\hline
\end{tabular}

\section{Conclusions}

We have introduced node-indri, a Node.js module to enable users with a good web development background (but minimal $\mathrm{C}++$ knowledge) to efficiently implement search applications. We have described how node-indri exposes Indri's functionalities and how it is currently being used in the backend of a largescale collaborative search system, that has been successfully tested with several hundred users. Furthermore, we compared node-indri's efficiency in the batch setting with Indri's and Pyndri's.

Acknowledgements: This work was funded by NWO projects LACrOSSE (612.001.605) and SearchX (639.022.722). The authors would like to thank Harry Scells for his input.

\footnotetext{
${ }^{5}$ https://trec.nist.gov/data/million.query07.html

${ }^{6}$ More details and benchmark code are included in the GitHub repository.
} 


\section{References}

1. Strohman, T., Metzler, D., Turtle, H., Croft, W.B.: Indri: A language model-based search engine for complex queries. In: ICIA. (2005)

2. Ounis, I., Amati, G., Plachouras, V., He, B., Macdonald, C., Lioma, C.: Terrier: A high performance and scalable information retrieval platform. In: OSIR

3. Yang, P., Fang, H., Lin, J.: Anserini: Enabling the use of lucene for information retrieval research. In: SIGIR. (2017)

4. Macdonald, C.: Combining terrier with apache spark to create agile experimental information retrieval pipelines. In: SIGIR. (2018)

5. Van Gysel, C., Kanoulas, E., de Rijke, M.: Pyndri: a python interface to the indri search engine. In: ECIR. (2017)

6. Tilkov, S., Vinoski, S.: Node. js: Using javascript to build high-performance network programs. IEEE Internet Computing (2010)

7. Lavrenko, V., Croft, W.B.: Relevance based language models. In: SIGIR. (2001)

8. Putra, S.R., Moraes, F., Hauff, C.: Searchx: Empowering collaborative search research. In: SIGIR. (2018) 